\title{
THE USE OF KAINIC ACID FOR STUDYING THE ORIGINS OF SCALP-RECORDED AUDITORY BRAINSTEM RESPONSES IN THE GUINEA PIG
}

\author{
JOHN N. GARDI and SANFORD C. BLEDSOE, Jr.* \\ Coleman Laboratory, 863-HSE, University of California School of Medicine, San Francisco, CA 94143 \\ (U.S.A.) \\ and \\ *Kresge Hearing Research Institute, The University of Michigan, Ann Arbor, MI 48109 (U.S.A.)
}

(Received May 27th, 1981; Revised version received and Accepted June 25th, 1981)

\begin{abstract}
Kainic acid was injected into the medial nucleus of the trapezoid body (MNTB) of guinea pigs to evaluate its use in studying generator loci of the scalp-recorded auditory brain stem response (ABR). Sound-evoked near-field potentials from the MNTB and far-field ABRs were recorded before, during and up to $2 \mathrm{~h}$ after the injections. Two hours post-injection, small amounts of kainic acid $(0.25 \mathrm{nmol}$ in $0.1 \mu$ l of Ringer solution) resulted in neuronal destruction which histologically appeared confined to the MNTB. Larger amounts $(10 \mathrm{nmol}$ in $1.0 \mu \mathrm{l})$ produced more extensive lesions. Regardless of the dose of kainic acid, near-field activity evoked by contralateral ear stimulation was almost totally abolished and ABR wave III amplitude was reduced by as much as $60 \%$. In future studies, the use of excitotoxic amino acids to produce lesions within complex nuclear subdivisions of the auditory pathway may yield valuable information as to the relative contributions that brainstem structures make to the various waves comprising the ABR and about the behavioral effects that axon sparing lesions produce.
\end{abstract}

The ability to selectively destroy neuronal cell bodies while preserving axons of passage has been a persistent need for investigations in many areas of neuroscience. In the brainstem of the mammalian auditory system, complex arrangements of cell somata and axons of passage are encountered at several levels, including: the cochlear nuclei, the superior olivary complex and the nuclei and tracts of the lateral lemniscus. Evidence obtained by ablating many of these structures with traditional techniques has provided some insight into the origins of the auditory brainstem response $(\mathrm{ABR})$; a series of synchronous waves recorded at the scalp during the first few milliseconds following abrupt acoustic stimuli $[1,4,10,15]$. However, more precise information as to the neurons generating the ABR is needed before its full potential as a clinical diagnostic tool can be realized.

Recently, much attention has been given to the excitatory and neurotoxic actions of kainic acid $[2,3,5,12]$. When appropriately injected into the brain, this potent neurotoxin causes the destruction of neuronal cell bodies and dendrites while 


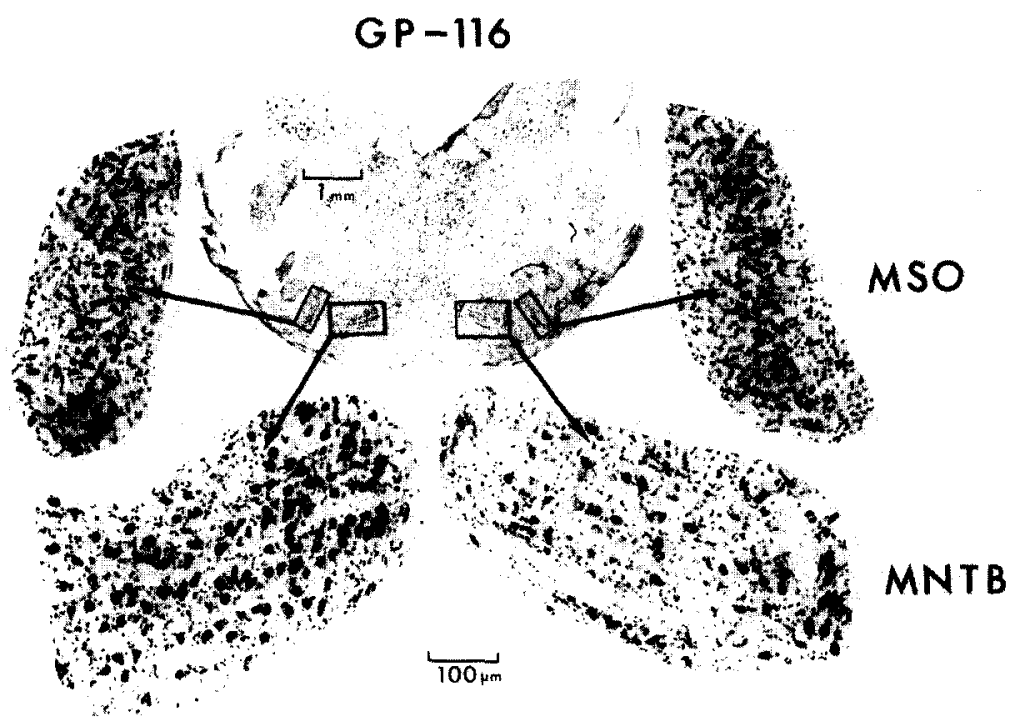

GP-109

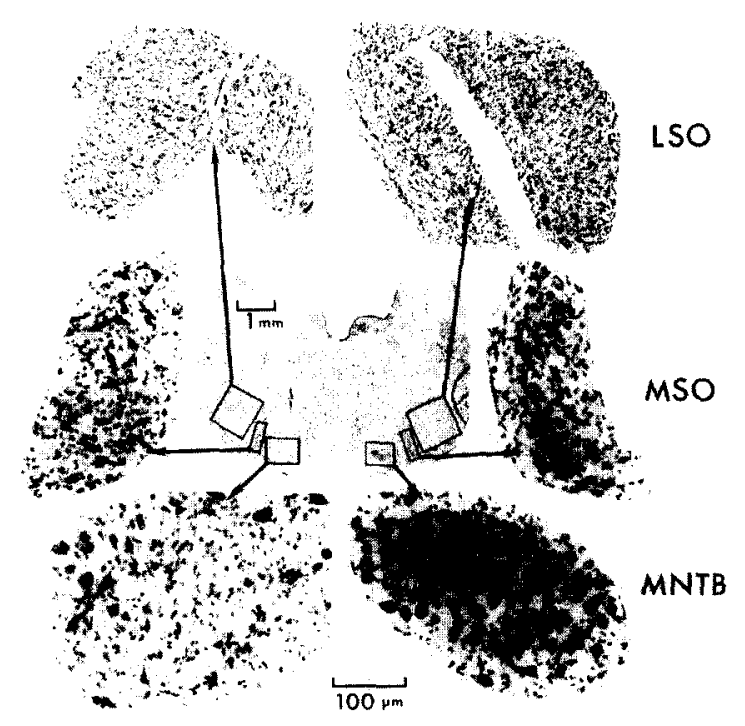

Fig. 1. Coronal section through the guinea pig superior olivary complex $2 \mathrm{~h}$ after kainic acid injection $(0.25 \mathrm{nmol}$ in $0.1 \mu \mathrm{l}$ Ringer solution) into the right medial nucleus of the trapezoid body (MNTB). As a control, Ringer solution $(0.1 \mu \mathrm{l})$ was injected into left MNTB. At higher magnification of the boxed regions, it is evident that neuronal cell bodies in the right MNTB are grossly crenulated, pyknotic, shrunken, and/or disrupted, whereas those in the left MNTB are normal in shape and number. Note that with this small dose of kainic acid, neurons in the right medial superior olive (MSO) adjacent to the injection site appear unaffected. $40 \mu \mathrm{m}$ section; cresyl violet stain. 
sparing axons of passage and nerve terminals of extrinsic origin [5]. The present study was undertaken to ascertain the usefulness of kainic acid in elucidating the brainstem elements generating the various waves comprising the ABR. The experimental approach was to inject small amounts of the neurotoxin into the medial nucleus of the trapezoid body (MNTB), a subdivision of the superior olivary complex, and evaluate the effects on sound-evoked near-field potentials within the MNTB and the far-field ABRs at the scalp. The extent of the resulting neuronal destruction was determined histologically.

Pigmented guinea pigs (220-370 g) of either sex were anesthetized with sodium pentobarbital (Nembutal, $25 \mathrm{mg} / \mathrm{kg}$, i.p.), paralyzed with gallamine triethiodide (Flaxedil, $20 \mathrm{mg} / \mathrm{kg}$, i.v.), and artificially respired. The skull was opened and the cerebellum aspirated to provide an unobstructed approach to the dorsal surface of the brainstem. To minimize mechanical damage, a microinjection apparatus was prepared by sealing the needle of a $1.0 \mu \mathrm{l}$ Hamilton syringe into a glass micropipette (50-80 $\mu \mathrm{m}$ tip, o.d.) filled with the solution to be injected. A silver wire was coupled to the needle to record near-field evoked potentials from the injection site. The micropipette was stereotaxically placed in the MNTB with guidance from direct visual sightings off prominent dorsal surface landmarks and near-field potentials which easily distinguish the subdivisions of the superior olivary complex [6, 14]. The tip of the pipette was lowered to a depth $(4.5-5.0 \mathrm{~mm})$ were maximum amplitude of the field potential in the MNTB was evoked by monaural stimulation of the contralateral ear. Kainic acid $(0.25-10.0 \mathrm{nmol}$ in $0.1-1.0 \mu \mathrm{l}$ of Ringer solution [11], $\mathrm{pH}$ 7.6) was infused over a 2 min period and the pipette left in place an additional $5 \mathrm{~min}$ before removal. Control injections of an equal volume of Ringer solution alone were made in the opposite MNTB prior to each injection of kainic acid. In this way, both control and experimental manipulations were made in the same preparation.

Three cycles of a $10.0 \mathrm{kHz}$ tone pip served as the acoustic stimulus. The ABRs were recorded as the potential difference between needle electrodes inserted under the skin at the vertex (active) and ipsilateral mastoid (reference); another needle at the contralateral mastoid provided ground. ABR waveforms were obtained prior to surgery, after surgical exposure, and just before, during and up to $2 \mathrm{~h}$ following injections of Ringer solution or kainic acid in Ringer solution. Two hours after injecting kainic acid, the animals were perfused intracardially with saline followed by $10 \%$ neutral buffered formaline. Frozen sections were cut in a coronal plane at $40 \mu \mathrm{m}$ and stained for histological examination with cresyl violet.

Fig. 2. Coronal section through the guinea pig superior olivary complex $2 \mathrm{~h}$ after a large dose of kainic acid $(10 \mathrm{nmol}$ in $1.0 \mu \mathrm{l}$ ) was injected into left medial nucleus of the trapezoid body (MNTB). Ringer solution $(1.0 \mu \mathrm{l})$ was injected the right MNTB. At higher manification of the boxed regions, it can be seen that with this dose of kainic acid the destruction has spread to include portions of the medial superior olive (MSO) adjacent to the injection site. However, the lateral superior olive (LSO) on the side injected with kainic acid appears unaffected. Note the near total destruction of cells in the left MNTB; this contrasts with the amount of visible cellular material in the lesioned MNTB shown in Fig. 1. $40 \mu \mathrm{m}$ section; cresyl violet stain. 
The effect of injecting Ringer solution $(0.1 \mu \mathrm{l})$ and kainic acid $(0.25 \mathrm{nmol}$ in 0.1 $\mu \mathrm{l})$ into the MNTB are illustrated in a mid-olivary coronal section in Fig. 1. At low magnification, the pale and grossly abnormal appearance of the cells in the right MNTB (kainic acid) contrasts with the normal intense staining of the cells in the left MNTB (Ringer solution). In this example, the rostral-caudal extent of the neuronal lesion produced by kainic acid was of the order of $300-400 \mu \mathrm{m}$, or about one-third to one-half the total rostral-caudal distance of the MNTB. At higher magnification, few remaining cells in the right MNTB appear grossly crenulated, pyknotic, shrunken and/or disrupted. In contrast, cells in the left MNTB seemed normal in shape and number with the staining of Nissl material discrete and intense. The normal appearance of the adjacent medial superior olive (MSO) on both the control and experimentally lesioned side indicates that with this small dose of kainic acid neuronal destruction is confined to the MNTB injection site.

As expected, larger amounts of kainic acid as well as larger injection volumes produced more extensive damage. A representative example of an animal injected with $10 \mathrm{nmol}$ of kainic acid in a $1.0 \mu \mathrm{l}$ volume is shown in Fig. 2. Destruction within the MNTB which received kainic acid is now more complete and the lesion extends to include portions of the MSO, but not the lateral superior olive (LSO).

It is well known that the large principal cells in the MNTB receive a monosynaptic excitatory input from neurons in the contralateral ventral cochlear nucleus and the near-field potentials in the MNTB are largest to monoaural contralateral stimulation $[6,9,13,14]$. In evaluating the electrophysiological effects of kainic acid, near-field potentials in response to ipsilateral and contralateral ear stimulation were obtained before and after control and experimental injections of Ringer solution and kainic acid, respectively. Fig. 3 shows results from an animal in which 0.25 nmol of kainic acid in $0.1 \mu$ l Ringer solution were injected into the right MNTB (see Fig. 1). Ringer solution $(0.1 \mu \mathrm{l})$ in the left MNTB failed to alter near-field activity evoked by right or left ear stimulation. However, with the electrode in the right MNTB, there was a marked decrement in local activity to contralateral left ear stimulation after the injection of kainic acid.

Fig. 4 illustrates the effect of kainic acid on the scalp-recorded ABR. The waveforms were obtained pre- and post-surgical exposure, after injecting Ringer solution, after injecting kainic acid, and $2 \mathrm{~h}$ after the injection of kainic acid. The surgical exposure and Ringer solution in the left MNTB produced little change in the $\mathrm{ABR}$. However, kainic acid $(0.25 \mathrm{nmol}$ in $0.1 \mu \mathrm{l}$ Ringer solution) injected into the right MNTB caused pronounced and specific amplitude reductions in the ABR to contralateral left ear stimulation. These amplitude reductions were restricted to time segments coincident with ABR waves III and IV. Waves I and II were unaffected, indicating normal function of the cochlea, eighth nerve and presumably cochlear nuclear complex $[1,4,8,10,15]$. Two hours after injecting this small dose of kainic acid, ABR wave III and IV amplitudes were still reduced and the extent of the lesion appeared confined to the right MNTB (see Fig. 1). 

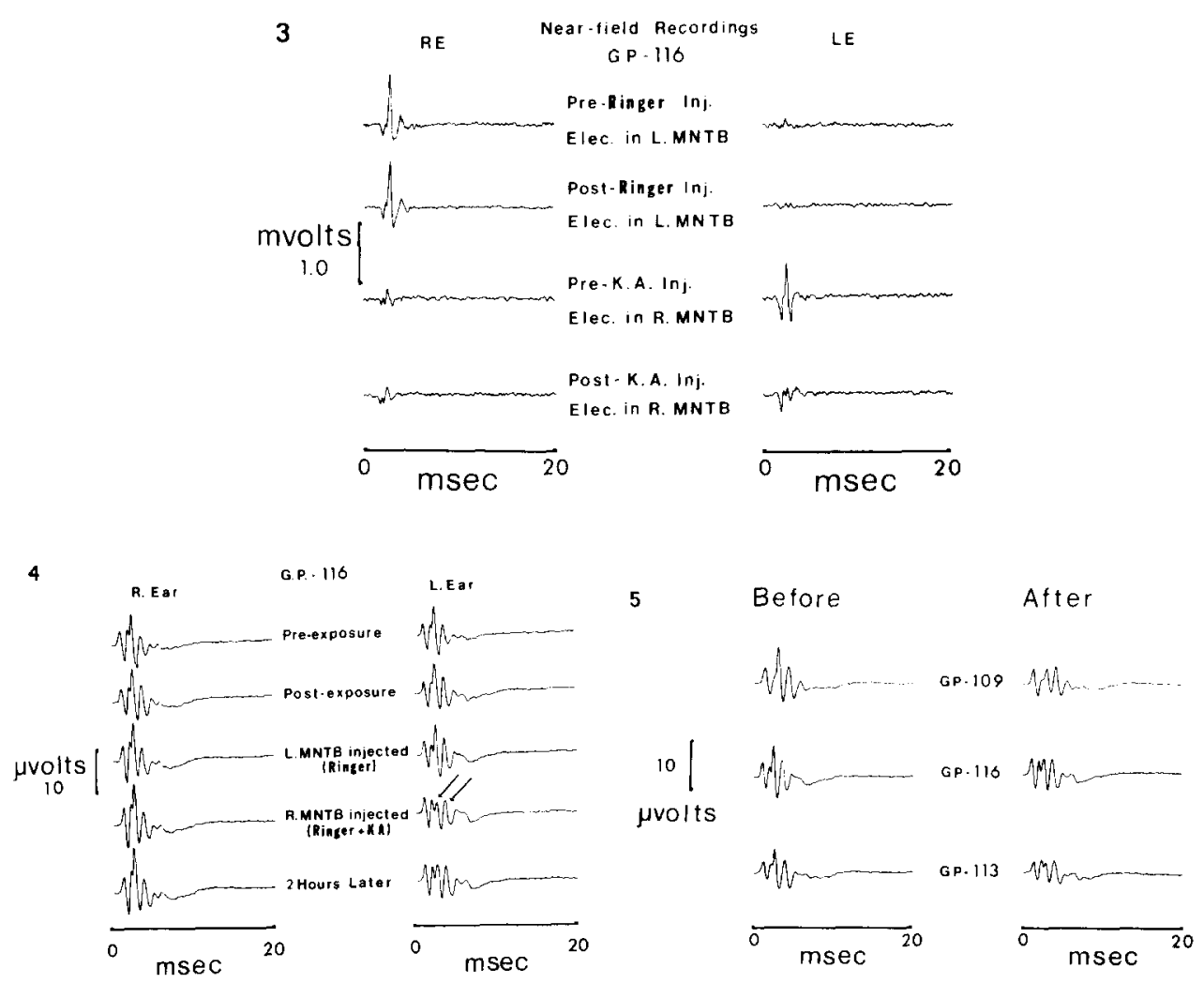

Fig. 3. Effects of Ringer solution and kainic acid on near-field evoked potentials recorded from the medial nucleus of the trapezoid body (MNTB). Ringer solution $(0.1 \mu \mathrm{l})$ injected into the left MNTB did not alter near-field activity evoked by contralateral right ear stimulation. However, immediately after injecting kainic acid $(0.25 \mathrm{nmol}$ in $0.1 \mu \mathrm{l}$ Ringer solution) into the right MNTB there was a marked decrement in near-field activity recorded from the injection site in response to contralateral left ear stimulation. The neuronal destruction observed $2 \mathrm{~h}$ after injecting kainic acid in this animal is shown in Fig. 1.

Fig. 4. Effects of kainic acid on scalp-recorded auditory brainstem response (ABR). ABRs in response to monaural right ear (left column) and left ear (right column) stimulation were recorded before surgical exposure of the brainstem; after surgical exposure; after injecting Ringer solution $(0.1 \mu \mathrm{l})$ into the left medial nucleus of the trapezoid body (MNTB); 5 min after injecting kainic acid $(0.25 \mathrm{nmol}$ in $0.1 \mu \mathrm{l}$ Ringer solution) into the right MNTB; and finally $2 \mathrm{~h}$ following the injection of kainic acid. The only profound change in the ABR was a marked decrease in the amplitudes of waves III and IV after injecting the right MNTB with kainic acid (see arrows). Effects of near-field potentials from this animal are shown in Fig. 3.

Fig. 5. ABR waveforms from 3 different guinea pigs, before and after kainic acid microinjection. Waveforms in the right column were obtained 5 min after injection; no additional changes occurred over a $2 \mathrm{~h}$ period following injection of kainic acid. 
In Fig. 5, results depicting the effects of kainic acid on the ABR from two additional guinea pigs are shown along with those of the previous example. It should be noted that GP-109 was injected with a large dose of kainic acid (see Fig. 2) but the results differ little from those of the other two animals. From all our findings the following generalizations for kainic acid injections can be made: (1) ABR wave III amplitude is markedly reduced (up to $60 \%$ in some cases); (2) ABR wave IV amplitude is less affected (decreasing by only as much as $25 \%$ in some cases); (3) ABR waves I and II remain virtually unaffected by kainic acid injections into the MNTB; (4) large and small doses of kainic acid which result in large and small lesions, respectively, have similar effects on the ABR.

It has been reported that ABR wave III completely disappeared following midsagittal section of the trapezoid body [7]. This fact, coupled with the results of the present investigation, suggests that ABR wave III in the guinea pig arises principally from the contralateral MNTB. This is further supported by the finding that doses of kainic acid which produced lesions extending into portions of the adjacent MSO failed to modify the results obtained with smaller doses which resulted in destruction confined to the MNTB. However, the possibility that the contralateral LSO and/or the ventral nucleus of the lateral lemniscus contribute to wave III cannot be ruled out entirely.

Overall, these results demonstrate that the use of kainic acid and perhaps other excitotoxic amino acids may yield valuable information about the generator loci of volume-conducted auditory potentials. With further improvements in controlling the size of lesions, it may be possible to resolve the relative contributions that complex nuclear subdivisions make to all the waves comprising the ABR. Furthermore, the technique would seem applicable to studying the behavioral effects produced by lesioning specific auditory nuclei or even cell types within nuclei. For instance, with suitable administration (e.g. iontophoresis), it may be possible to measure the behavioral effects of a lesion confined to the octopus cell region of the large spherical cell region of the cochlear nucleus.

This work was performed when the authors were Postdoctoral Fellows at the Kresge Hearing Research Laboratory of the South, and was supported by NIH Grants NS-06094 and NS-07058. Facilities were provided in part by the Kresge Foundation.

1 Achor, L. and Starr, A., Auditery brain stem responses in the cat. II. Effects of lesions, Electroenceph. clin. Neurophysiol., 48 (1980) 174-190.

2 Bird, S., Ciulley, R., Wenthold, R. and Fex, J., Kainic acid injections result in degeneration of cochlear nucleus cells innervated by the auditory nerve, Science, 202 (1978) 1087-1089.

3 Bledsoe, S.C., Jr., Bobbin, R.P. and Chihal, D.M., Kainic acid: an evaluation of its action on cochlear potentials, Hear. Res., 4 (1981) 109-120.

4 Buchwald, J. and Huang, C., Far-field acoustic response: origins in the cat, Science, 189 (1975) $382-384$. 
5 Coyle, J., Mollivar, M. and Kuhar, M., In situ injection of kainic acid: A new method for selectively lesioning neuronal cell bodies while sparing axons of passage, J. comp. Neurol., 180 (1978) 301-324.

6 Galambos, R., Schwartzkopff, J. and Rupert, A., Microelectrode study of superior olivary nuclei, Amer. J. Physiol., 197 (1959) 527-536.

7 Gardi, J. and Berlin, C., Binaural interaction components of the guinea pig ABR: possible origins, Arch. Otolaryng., 107 (1981) 164-168.

8 Gardi, J., Merzenich, M. and McKean, C., Origins of the scalp-recorded frequency-following response in the cat, Audiology, 18 (1979) 353-381.

9 Guinan, J.C., Jr., Norris, B.E. and Guinan, S.S., Single auditory units in the superior olivary complex. II: Locations of unit categories and tonotopic organization, Int. J. Neurosci., 4 (1972) 147-166.

10 Goldenberg, R. and Derbyshire, A., Averaged evoked potentials in cats with lesions of the auditory pathway, J. Speech Hear. Res., 18 (1975) 420-429.

11 Konishi, T., Action of tubocurarine and atropine on the crossed olivocochlear bundle, Acta otolaryng. (Stockh.), 74 (1972) 252-264.

12 Masterton, R., Glendenning, K. and Hutson, K., Preservation of trapezoid body fibers after biochemical ablation of superior olives with kainic acid, Brain Res., 173 (1979) 156-159.

13 Morest, D.K., The collateral system of the medial nucleus of the trapezoid body of the cat, its neuronal architecture and relation to the olivo-cochlear bundle, Brain Res., 9 (1968) 288-311.

14 Moushegian, G., Rupert, A. and Whitcomb, M.A., Medial superior-olivary-unit response patterns to monaural and binaural clicks, J. Acoust. Soc. Amer., 36 (1964) 196-202.

15 Starr, A. and Hamilton, A., Correlation between confirmed sites of neurological lesions and abnormalities of far-field auditory brainstem responses, Electroenceph. clin. Neurophysiol., 41 (1976) 595-608. 